\title{
SIX NEW SETS OF INDEPENDENT AXIOMS FOR DISTRIBUTIVE LATTICES WITH $O$ AND $I$
}

\section{BOLESŁAW SOBOCIŃSKI}

In [4] Grau defined and discussed the following ternary Boolean functor*

$A \quad \phi(a b c)=(a \cap b) \cup(b \cap c) \cup(c \cap a)$

which, since the formula

$B \quad(a \cap b) \cup(b \cap c) \cup(c \cap a)=(a \cup b) \cap(b \cup c) \cap(c \cup a)$

holds in Boolean algebra, is, obviously, the self-dual operation.

In [1] Birkhoff and Kiss have shown that, if this connective of Grau is considered as lattice operation (called the median of $a, b, c)$, then a distributive lattice with $O$ and $I$ can be defined in terms of this single functor. This result is formulated in [2], pp. 137-138, theorem 4, as follows

Let $\mathbf{A}$ be any algebric system with a ternary operation $\phi\left(\begin{array}{lll}a & b & c\end{array}\right)$ and elements $O$ and I such that it satisfies

(i) $\phi(O a I)=a$

(ii) $\phi\left(\begin{array}{lll}a & b & a\end{array}\right)=a$

(iii) $\phi(a b c)=\phi(b a c)=\phi(b c a)$

(iiv) $\phi(\phi(a b c) d e)=\phi(\phi(a d e) b \phi(c d e))$

identically. Then if we define

(ï) $\quad a \cup b=\phi(a I b)$ and $a \cap b=\phi(a O b)$

A is a distributive lattice in which $A$ bolds.

As problem 64, in [2], p. 138, Birkhoff put the question whether at least part of (iii) can be dispensed with, if a suitable permutation of (ï̈) is

\footnotetext{
*Instead of Birkhoff's notation for this ternary operation: $\left(\begin{array}{lll}a & b & c\end{array}\right)$, cf. e.g. [2], p. 137, I use the symbol: $\phi(a b c)$ throughout this paper.
} 
used. The various solutions to this problem are already published by several authors. Namely:

a) In [7] Vassiliou has proved that conditions (i) - (ï̈) of Birkhoff follow from (i), (ii) and the following formula

(ivi) $\quad \phi(d \phi(a b c) e)=\phi(\phi(e d c) b \phi(e d a))$

b) In [3], pp. 24-25, Croisot has proved that (i), (ii) and

(

imply (iii) and (ï̈).

c) In [5], p. 49, Hashimito has shown that we can deduce (iii) and (ï̈) from (i), (ii) and

( $\ddot{\mathrm{viii}}) \phi\left(d \phi\left(\begin{array}{lll}a & b & c\end{array}\right) e\right)=\phi(\phi(e b d) a \phi(e c d))$

d) In [6], p. 30, Sholander announced without proof that conditions (i) - (i vi) follow from the following two formulas

$$
\phi(O a \phi(l \quad b l))=a
$$

and

( $\ddot{\mathbf{x}}) \quad \phi(d \phi(a b c) e)=\phi(\phi(d b e) c \phi(a d e))$

Many other axiom-systems satisfying Birkhoff's problem for distributive lattices with $O$ and $I$ can be established and added in this list. I present here six such sets of postulates. These axiom-systems possess a certain common feature, since the same permutation of (ivi) is involved in their construction. Namely, I shall show that

a) Conditions (iii) and (iï) follow from (i), (ii) and

(

b) Each of the following formulas

(巛̈ii) $\phi(O \phi(b a a) I)=a$

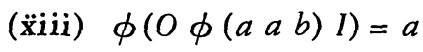

and

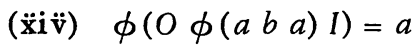

together with ( $\ddot{x i})$ implies (i) and (ii).

c) Conditions (i) and (̈i) follow from (ii) and either

$(\ddot{\mathbf{x}} \ddot{\mathrm{v}}) \quad \phi(O \phi(d \phi(a b c) e) I)=\phi(\phi(d c e) \phi(d a e) b)$

or

$(\dddot{\mathrm{x}} \ddot{\mathrm{v} i}) \quad \phi(d \phi(a b c) e)=\phi(O \phi(\phi(d c e) \phi(d a e) b) I)$ 
Proof:

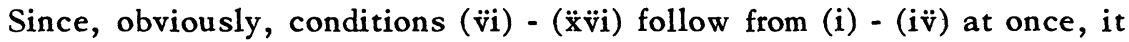
is sufficient to prove that the latter formulas follow from the respective sets of postulates mentioned in $a)-c)$. Hence:

$\S 1$. Assume conditions (i), (ii) and (艹̈i), i.e. the formulas

$$
\begin{aligned}
& \text { A1 } \quad \phi\left(\begin{array}{lll}
O & a & I
\end{array}\right)=a \\
& \text { A2 } \phi\left(\begin{array}{lll}
a & b & a
\end{array}\right)=a \\
& \text { A3 } \phi(d \phi(a b c) e)=\phi(\phi(d c e) \phi(d a e) b)
\end{aligned}
$$

Then:

$$
\begin{array}{ll}
\text { A4 } & \phi\left(\begin{array}{lll}
a & b & c
\end{array}\right)=\phi\left(\begin{array}{lll}
c & a & b
\end{array}\right) \\
A 5 & \phi\left(\begin{array}{lll}
a & b & c
\end{array}\right)=\phi\left(\begin{array}{lll}
b & c & a
\end{array}\right) \\
A 6 & \phi\left(\begin{array}{lll}
a & a & b
\end{array}\right)=a \\
A 7 & \phi\left(\begin{array}{lll}
b & a & a
\end{array}\right)=a \\
\text { A8 } & \phi\left(\begin{array}{lll}
a & b & c
\end{array}\right)=\phi\left(\begin{array}{lll}
b & a & c
\end{array}\right)
\end{array}
$$$$
[A 3, d / O, e / I ; A 1, a / \phi(a b c) ; A 1, a / c ; A 1]
$$$$
[A 4 ; A 4, a / c, b / a, c / b]
$$$$
[A 2 ; A 4, c / a]
$$$$
[A 2 ; A 5, c / a]
$$

Dem.: $\phi(a b c)=\phi(a \phi(b b a) c)=\phi(\phi(a a c) \phi(a b c) b)=\phi(a \phi(a b c) b)$ $=\phi\left(\phi\left(\begin{array}{lll}a & c & b\end{array}\right) \phi\left(\begin{array}{lll}a & a & b\end{array}\right) b\right)=\phi\left(\phi\left(\begin{array}{lll}a & c & b\end{array}\right) a b\right)=\phi\left(b \phi\left(\begin{array}{lll}a & c & b\end{array}\right) a\right)=$ $\phi\left(\phi\left(\begin{array}{lll}b & b & a\end{array}\right) \phi\left(\begin{array}{lll}b & a & a\end{array}\right) c\right)=\phi\left(\begin{array}{lll}b & a & c\end{array}\right)$

$[A 6, a / b, b / a ; A 3, a / b, c / a, d / a, e / c ; A 6, b / c ; A 3, d / a, c / b ; A 6$; $A 4, a / \phi(a c b), b / a, c / b ; A 3, b / c, c / b, d / b, e / a ; A 6, a / b, b / a ; A 7]$

$$
\phi(\phi(a b c) d e)=\phi(\phi(a d e) b \phi(c d e))
$$

Dem.: $\phi\left(\phi\left(\begin{array}{lll}a & b & c\end{array}\right) d e\right)=\phi\left(\begin{array}{lll}d & \phi & \left.\left(\begin{array}{lll}a & b & c\end{array}\right) e\right)=\phi\left(\phi\left(\begin{array}{lll}d & c & e\end{array}\right) \phi(d a e) b\right.\end{array}\right)=$ $\phi(\phi(d a e) b \phi(d c e))=\phi(\phi(a d e) b \phi(c d e))$

$[A 8, a / \phi(a b c), b / d, c / e ; A 3 ; A 5, a / \phi(d c e), b / \phi(d a e), c / b ; A 8$, $a / d, b / a, c / e ; A 8, a / d, b / c, c / e]$

Since $A 8, A 5$ and $A 9$ constitute conditions (iii) and (i $\ddot{v})$, the proof is completed. The following modification of Croisot's argumentation, given in [3], pp. 24-25, shows that the axioms $A 1-A 3$ are mutually independent:

$\alpha)$ Assume that both $O$ and $I$ are Boolean $O$ and 1 respectively and that $\phi\left(\begin{array}{lll}a & b & c\end{array}\right)$ is the Boolean formula such that $\phi\left(\begin{array}{lll}a & b & c\end{array}\right)=a$. Then $A 2$ and $A 3$ are verified, but $A 1$ becomes a false formula.

$\beta$ ) Assume that both $O$ and $I$ are Boolean 0 and that $\phi(a b c)$ is the Boolean formula: $a \cup b \cup c$. Then $A 1$ and $A 3$ are verified, but $A 2$ is falsified.

$\gamma$ ) Assume that both $O$ and $I$ are Boolean $O$ and $I$ respectively and that $\phi(a b c)$ is the Boolean formula: $(a \cup b) \cap c$. Then $A 1$ and $A 2$ are verified, but $A 3$ is a false Boolean formula.

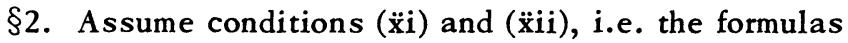

$B 1 \phi(d \phi(a b c) e)=\phi(\phi(d c e) \phi(d a c) b)$ 
and

$B 2 \phi\left(O \phi\left(\begin{array}{lll}b & a & a\end{array}\right) I\right)=a$

Then:

B3 $\phi(b a a)=\phi(a c \phi(b a r))$

Dem.: $\phi(b a a)=\phi(O \phi(\phi(b c c) \phi(b a a) \phi(b a a)) I)=\phi(\phi(O \phi(b a a) I)$ $\phi(O \phi(b c c) I) \phi(b a a))=\phi(a c \phi(b a a))$

$[B 2 ; a / \phi(b a a), b / \phi(b c c) ; B 1, a / \phi(b c c), b / \phi(b a a), c / \phi(b a a)$, $d / O, e / I ; B 2 ; B 2, a / c]$

$B 4 \phi(b a a)=\phi(\phi(b a a) a c)$

Dem.: $\phi(b a a)=\phi(O \phi(b \phi(b a a) \phi(b a r)) I)=\phi(O \phi(\phi(b a a) c \phi(b \phi$ $\left.\left.\left.\left(\begin{array}{ll}b & a\end{array} a\right) \phi(b a a)\right)\right) I\right)=\phi(\phi(O \phi(b \phi(b a a) \phi(b a a)) I) \phi(O \phi(b a a)$ I) $c)=\phi\left(\phi\left(\begin{array}{lll}b & a & a\end{array}\right) a c\right)$

$\left[B 2, a / \phi(b a a) ; B 3, a / \phi(b a r) ; B 1, a / \phi\left(\begin{array}{lll}b & a & a\end{array}\right), b / c, c / \phi(b \phi(b a r)\right.$ $\phi(b a a)), d / O, e / I ; B 2, a / \phi(b a a) ; B 2]$

B5

$a=\phi\left(\begin{array}{lll}b & a & a\end{array}\right)$

Dem.: $\quad a=\phi\left(O \phi\left(\begin{array}{lll}b & a & a\end{array}\right) I\right)=\phi(O \phi(\phi(b a a) a \phi(b b b)) I)=\phi(\phi(O \phi(b b b)$ I) $\phi(O \phi(b a a) I) a)=\phi(b a a)$

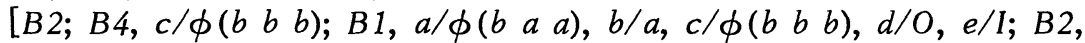
$a / b ; B 2]$

B6

$\phi(O \quad a I)=a$

$[B 2 ; B 5]$

B7 $a=\phi\left(\begin{array}{lll}a & b & a\end{array}\right)$

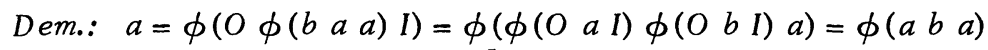

$[B 2 ; B 1, a / b, b / a, c / a, d / O, e / I ; B 6 ; B 6, a / b]$

Since we obtained $B 6$ and $B 7$, i.e. conditions (i) and (ii), the present set of postulates implies the axiom-system discussed in $\S 1$. Hence, the proof is given. The first and the third interpretations given in $\S 1$ show that $B 1$ and $B 2$ are mutually independent.

$\S 3$. Assume now conditions ( $\ddot{x} i)$ and ( $\ddot{x} i i i)$, i.e. the formulas

$C 1$ $\phi(d \phi(a b c) e)=\phi(\phi(d c e) \phi(d a e) b)$

and

$C 2 \phi(O \phi(a a b) I)=a$

Then:

C3 $\phi(a a b)=\phi(c a \phi(a a b))$

Dem.: $\phi(a a b)=\phi(O \phi(\phi(a a b) \phi(a a b) \phi(c c b)) I)=\phi(\phi .(O \phi(c c b) I)$ $\phi(O \phi(a a b) I) \phi(a a b))=\phi(c a \phi(a a b))$

$[C 2, a / \phi(a a b), b / \phi(c c b) ; C 1, a / \phi(a a b), b / \phi(a a b), c / \phi(c c b)$, $d / O, e / I ; C 2, a / c ; C 2]$. 
Dem.: $a=\phi(O \phi(a a b) I)=\phi(O \phi(b b b) a \phi(a a b)) I)=\phi(\phi(O \phi(a a b) I)$ $\phi(O \phi(b b b) I) a)=\phi(a b a)$

$[C 2 ; C 3, c / \phi(b \quad b \quad b) ; C 1, a / \phi(b \quad b \quad b), b / a, c / \phi(a a b), d / O, e / l ; C 2 ;$

C5

$a=\phi\left(\begin{array}{lll}a & a & b\end{array}\right)$

Dem.: $a=\phi\left(a \phi\left(\begin{array}{lll}b & b & b\end{array}\right) a\right)=\phi(\phi(\phi(a b a) \phi(a b a) b))=\phi(a a b)$

$[C 4, b / \phi(b b b) ; C 1, a / b, c / b, d / a, e / a ; C 4 ; C 4]$

C6 $\phi(O$ a $I)=a$

[C2;C5]

Since $C 6, C 4$ and $C 1$ constitute the axiom-system given in $\S 1$, the proof is completed. The first interpretation presented in $\S 1$ proves that $C 2$ does not follow from $C 1$. Assume now that

$\delta)$ Both $O$ and $I$ are Boolean 0 and 1 respectively and $\phi\left(\begin{array}{lll}a & b\end{array}\right)$ is the Boolean formula such that $\phi(a b c)=b$.

This interpretation shows that $C 2$ does not imply $C 1$.

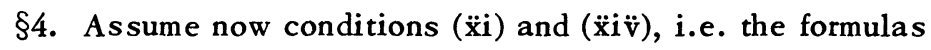

$D 1 \quad \phi(d \phi(a b c) e)=\phi(\phi(d c e) \phi(d a e) b)$

and

D2 $\phi\left(O \phi\left(\begin{array}{lll}a & b & a\end{array}\right) I\right)=a$

Then:

D3 $\phi\left(\begin{array}{lll}a & b & a\end{array}\right)=\phi(a a b)$

Dem.: $\phi\left(\begin{array}{lll}a & b & a\end{array}\right)=\phi\left(O \phi\left(\phi\left(\begin{array}{lll}a & b & a\end{array}\right) b \phi(a b a)\right) I\right)=\phi(\phi(O \phi(a b a) I) \phi(O$ $\phi(a b a) I) b)=\phi(a a b)$

$[D 2, a / \phi(a b a) ; D 1, a / \phi(a b a), c / \phi(a b a), d / O, C / I ; D 2: D 2]$

D4 $\phi(O \phi(a a b) I)=a$

$[D 2 ; D 3]$

Since we obtained $D 4$, we have the axiom-system presented in $\S 3$, and, therefore, the proof is given. The first and the third interpretations from $\S 1$ show that $D 1$ and $D 2$ are mutually independent.

$\S 5$. Assume conditions (ii) and ( $\ddot{x} \ddot{v})$, i.e. the formulas

E1 $\phi\left(\begin{array}{lll}a & b & a\end{array}\right)=a$

and

E2 $\phi\left(O \phi\left(d \phi\left(\begin{array}{lll}a b & c) e\end{array}\right)=\phi(\phi(d c e) \phi(d a e) b)\right.\right.$

Then:

E3 $\phi(O$ a $I)=a$ 
Dem.: $\phi(O a I)=\phi(O \phi(a \phi(a a r) a) I)=\phi(\phi(a r a) \phi(a r a) a)=\phi(a a r)$ $=a$

$[E 1, b / \phi(a a a) ; E 2, b / a, c / a, d / a, e / a ; E 1, b / a ; E 1, b / a ; E 1, b / a]$

$E 4 \phi(d \phi(a b c) e)=\phi(\phi(d c e) \phi(d a e) b) \quad[E 2 ; E 3, a / \phi(d \phi(a b c) e)]$

Since we proved $E 3$ and $E 4$, we obtained the set of postulates given in $\S 1$. Hence, the proof is completed. The first and the second interpretations ırom $\S 1$ show that $E 1$ and $E 2$ are mutually independent.

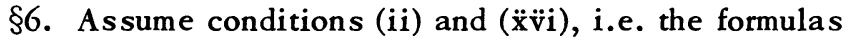

F1 $\quad \phi\left(\begin{array}{lll}a & b & a\end{array}\right)=a$

and

F2 $\phi(d \phi(a b c) e)=\phi(O \phi(\phi(d c e) \phi(d a e) b) I)$

Then:

F3 $a=\left(\begin{array}{lll}O & a & I\end{array}\right)$

Dem.: $a=\phi(a \phi(a a r) a)=\phi\left(O \phi\left(\phi\left(\begin{array}{lll}a & a & a\end{array}\right) \phi(a a a) a\right) I\right)=\phi(O \phi(a a a)$ $I)=\phi(O a I)$

$[F 1, b / \phi(a a a) ; F 2, b / a, c / a, d / a, e / a ; F 1, b / a ; F 1, b / a ; F 1, b / a]$

F4 $\quad \phi(d \phi(a b c) e)=\phi(\phi(d c e) \phi(d a e) b)$

$[F 2 ; F 3, a / \phi(\phi(d c e) \phi(d a e) b)]$

Since $F 3$ and $F 4$ are obtained, we have the axiom-system given in $\$ 1$. Therefore, the proof is completed. The same interpretations which are used in $\$ 5$ show that $F 1$ and $F 2$ are also mutually independent.

\section{BIBLIOGRAPHY}

[1] Garret Birkhoff and S. A. Kiss: A ternary operation in distributive lattices. Bulletin of the American Mathematical Society, v. 53 (1947), pp. 749-752.

[2] Garrett Birkhoff: Lattice Theory. Revised Edition. American Mathematical Society Colloquium Publications, v. XXV. New York City, 1948.

[3] R. Croisot: Axiomatique des lattices distributives. Canadian Journal of Mathematics, v. III (1951), pp. 24-27.

[4] A. A. Grau: Ternary Boolean algebra. Bulletin of the American Mathematical Society, v. 53 (1947), pp. 567-572.

[5] Junji Hashimoto: A Ternary Operation in Lattices. Mathematica Japonicae, v. II (1950-1952), pp. 49-52.

[6] Marlow Sholander: Postulates for distributive lattices. Canadian Journal of Mathematics, v. III (1951), pp. 28-30.

[7] Ph. Vassiliou: A set of postulates for distributive lattices. Publication de l' Université technique nationale d'Atbènes, No 5 (1950). 